\title{
Developing Pharmacotherapy for Cyberaddictions?
}

\author{
Sophia Achab ${ }^{*}$, Manuela Bertolini and Laurent Karila
}

Division of Addictology, Department of Mental Health and Psychiatry, University Hospitals of Geneva, Switzerland

\begin{abstract}
When developing a new pharmacotherapy for a given indication one has to differentiate between de novo developments and drug repurposing. The practice of drug repurposing can be based on 2 core concepts: (1) the Known compound-new target and (2) the Known target-new indication approach. Concerning cyberaddictions, the known targetnew indication approach becomes the most promising in consideration of different hypotheses classifying cyberaddiction within other well defined psychiatric disorders, for which efficacious pharmacotherapies have been corroborated. Supposing similar or identical neurobiological underpinnings, a common pharmacological target can be formulated as working hypothesis. Research on new pharmacotherapies for cyberaddiction can thus be conceptualized along different axes which follow the various current theorizations concerning the diagnosis categorization of cyberaddictions. As Internet addiction shares features which are in common with substance use disorders or obsessive-compulsive disorders, treatments found to be efficacious in these disorders may be promising development candidates for cyberaddiction. Also, cyberaddiction has been conceptualized to represent the corollary of other psychiatric disorders. Thus, currently mainly three research tracks can be distinguished: (a) the addictions track (b) the OCD track, and (c) the comorbidity track.
\end{abstract}

Keywords: Internet addiction, cyberaddiction, pharmacotherapy, treatment.

\section{INTRODUCTION}

The validity of the concept of cyberaddiction has been discussed since more than a decade. Despite an increasing acceptance of the concept, large number of publications underlying its prevalence and its burden on public health, potential pharmacotherapeutic approaches have only slightly been tried. This may be due to various reasons.

The consensus regarding the validity of the diagnosis may still not have reached enough momentum to motivate researchers and/or pharmaceutical companies in engaging what still may be considered a risky and possibly futile endeavor. Another discouragement may be the heterogeneous clinical phenomenology of cyberaddiction, as it may take numerous forms (e.g. cybersex, gambling, gaming, etc.) for which a common clinical and/or pharmacological target may seem intricate to found. Furthermore, traditional addiction treatment facilities have until now mainly been conceived to receive archetypal alcohol dependent or illegal drug dependent patients, and consequently may be not tailored to address this new clientele.

There may also still be a widely prevalent difficulty among professionals in the field to treat a primary psychologically manifesting phenomenon by a biological treatment. On the other side, the experience with pharmacotherapy in pathological gambling should oppose this argument. Finally, there is currently a rather critical atmosphere against the creation of new diagnosis, especially

*Address correspondence to this author at the Division of Addictology, Department of Mental Health and Psychiatry, University Hospitals of Geneva, Rue du grand-Pré, 70C, CH - 1202, Genève, Switzerland; Tel: + 41 22320 55; 50; Fax: + 412232028 40; E-mail: Sophia.Achab@hcuge.ch as the pharmaceutical industry has lately often been blamed for boosting the creation of new pharmacotherapy indications.

\section{ARGUMENTS AGAINST PHARMACOTHERAPY RESEARCH FOR CYBERADDICTION}

In consideration of the possible costs, the introduction of a new treatment, be it pharmacological or other, has to respond to possible critics.

The principal argument against the current development of pharmacotherapy research for cyberaddiction is possibly the insufficient validity of the cyberaddiction diagnosis. The scientific committee of the AMA has, underscored in its report on Internet dependence (http/www.ama-assn.org) the necessity to perform more empirical studies. To invest in the development of any form of therapy could therefore be considered by some to be risky. Various subtypes of cyberaddictions have been described. Young, for example, categorized Internet addiction into 5 types: Cybersex addiction; cyber relationship addiction, netcompulsion (e.g. gambling or shopping on the Internet), information overload (e.g. compulsive databases searching), and computer addiction (excessive game playing). The necessity/utility to treat these different phenomena, as well as their possible responsiveness to treatment may highly vary. Before launching treatment research programs, the cost-benefit ratio should be assessed. Currently, no longitudinal data is available on spontaneous evolutions of any form of cyberaddiction. Thus, even if cyberaddiction is a valid diagnosis entity with (temporary) burden of illness, treatment simply may be not necessary.

Another key argument against the introduction of the diagnosis category cyberaddiction is the same which has been affirmed for many of the recently introduced 
psychiatric diagnoses, i.e. the risk to further pathologize human existence.

Finally, one ethical inconvenience may derive from the fact that currently most patients are adolescents or early adults, a particularly sensible age concerning pharmacotherapeutic treatment, as publicized by the recent debates on antidepressant or stimulant treatments of children and adolescents.

\section{WHY PHARMACOTHERAPY FOR CYBERADDICT- ION SHOULD BE DEVELOPED?}

There are several reasons why pharmacotherapeutic approaches should be developed against cyberaddictions. Various epidemiological studies carried out in different countries presage a substantial burden of disease due to excessive use of Internet. There is increasing data linking Internet related addictive behaviors to neurobiological alterations which may become promising pharmacological targets.

On the other side, promising experiences with pharmacotherapy in other addictions may by extrapolation motivate research in this new field. Finally, pharmacotherapeutic research may boost addiction research in the field in general and especially regarding assessment instruments, they being diagnostic or measuring severity of respective chains.

\section{HOW TO DEVELOP PHARMACOTHERAPY?}

When developing a new pharmacotherapy for a given indication, different approaches can be differentiated (Fig. 1). One first classification can be made between "de novo developments" and "drug repurposing". Within the framework of a "de novo development", a drug - which is not marketed for any indication - progressed along the different development stages (chemical/biological design, preclinical, clinical phase I-III).

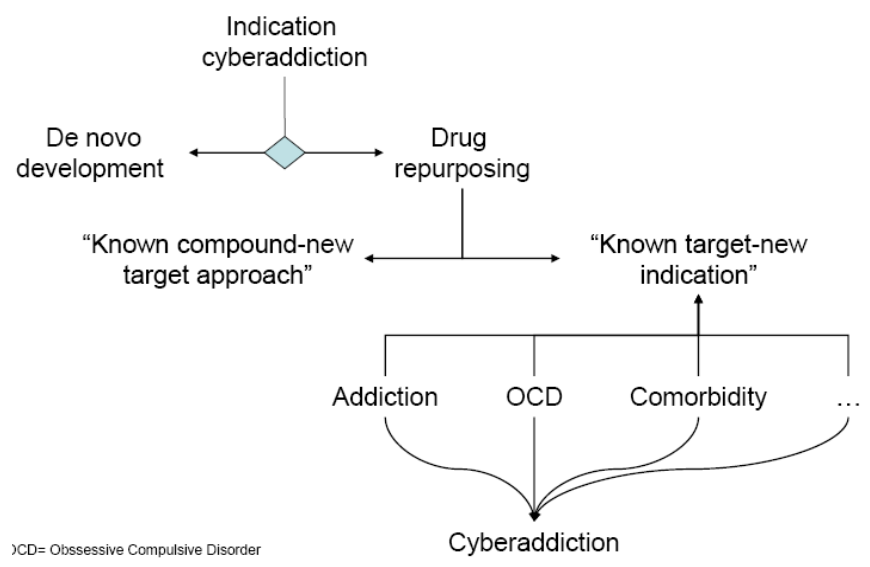

Fig. (1). Approaches for the development of new pharmacotherapies.

"Drug repurposing" (also known as drug repositioning, drug reprofiling, therapeutic switching or drug retasking) consists of the application of known drugs and compounds to new indications (i.e., new diseases).

A significant advantage of "drug repurposing" over "de novo drug development" is that in vitro and in vivo screening, chemical optimization, toxicology, bulk manufacturing, formulation development and even early clinical development have, in many cases, already been completed and can therefore be bypassed. Thus, the risk of failure for reasons of adverse toxicology may be reduced. Any newly identified drug use can thus be rapidly evaluated from phase II clinical trials on. In this way, drug developers can bypass almost $40 \%$ of the overall cost of bringing a drug to market [1]. As most of drugs fail during preclinical and clinical development [2] and this is the most significant reason for the high costs of pharmaceutical research and development, repositioning hence becomes a rather interesting method for pharmaceutical companies [3]. It may, however, also be of particular interest for physicians and their patients, as repurposed drugs can bypass much of the time needed to bring an efficacious drug to market.

The practice of drug repurposing can be based on 2 core concepts: (1) the Known compound-new target and (2) the Known target-new indication approach.

\section{KNOWN COMPOUND-NEW TARGET APPROACH}

The first approach is built on the fact that a particular drug can interact with multiple biological targets. Thus, this approach focuses on the identification of secondary so-called "off-target" drug actions, and then leads to the development of the drug in a new indication where the secondary target is relevant. The "off-target" in consequence becomes an "ontarget". Compounds with diverse off-target effects are often labeled "dirty" because of the side effects they induce. However, their undesirable side effect in one indication may sometimes provide a desirable effect in another indication. A "dirty drug" may then become a "desirably promiscuous drug".

\section{KNOWN TARGET-NEW INDICATION APPROACH}

This method is based on the concept that biological targets relevant to one disease may be involved also in other diseases as well. Thus, the aim of this second repurposing approach is to establish the relevance of a known drug target to a new disease.

A subtype of this known target-new indication approach may be the case when the biological substrate (e.g. a target protein) of the concerned diseases has not (sufficiently) been determined, but when the two diseases overlap largely in their symptomatology, they strongly suggest such a common biological substrate. This approach has been applied in the clinical development of selective serotonin reuptake inhibitors (SSRIs) for the various anxiety disorders.

Concerning cyberaddictions, the known target-new indication approach becomes the most promising in consideration of different hypotheses classifying cyberaddiction within other well defined psychiatric disorders, for which efficacious pharmacotherapies have been corroborated. Supposing similar or identical neurobiological underpinnings, a common pharmacological target can be formulated as working hypothesis.

\section{RESEARCH AXES FOR CYBERADDICTION}

Research on new pharmacotherapies for cyberaddiction can thus be conceptualized along different axes which follow the various current theorizations concerning the diagnosis categorization of cyberaddictions. As Internet addiction shares features which are common with substance use disorders or 
obsessive-compulsive disorders (OCD), treatments found to be efficacious in these disorders may be promising development candidates for cyberaddiction. Also, cyberaddiction has been conceptualized to represent the corollary of other psychiatric disorders. Thus, currently mainly three research tracks can be distinguished: (a) the addictions track (b) the OCD track, and (c) the comorbidity track.

\section{THE “ADDICTION TRACK"}

Cyberaddiction could be considered a part of the addiction spectrum: pharmacotherapy research should then ideally target common psychopathological phenomena of addictive behavior. Currently, the neurobiological systems focus would be the dopaminergic, opioid, GABAergic and glutamatergic systems.

Like substance abuse, problematic Internet use results in a dopaminergic search in the brain. There is for example evidence that Internet videogame playing is associated with dopamine release [4] in positron emission tomography (PET) taken during video game playing. Binding of Raclopride to dopamine receptors in the striatum was significantly reduced during the video game.

\section{Available Data}

While Naltrexone has the approval from the Food and Drug Administration for treating alcohol addiction, several published case reports have demonstrated its potential for treating pathological gambling, self injury, kleptomania and compulsive sexual behavior (e.g. [5]).

Bostwick and Bucci [6] have presented a case report of Naltrexone treatment which aimed to reduce compulsive Internet use for sexual gratification. They described a 24 years old male patient consulting for overconsumption of Internet pornography, who spent several hours every day chatting online, engaging in extended masturbation sessions and occasionally meeting cyber contacting people for spontaneous typically unprotected sex. The patient had been non-responsive to various therapeutic interventions over 7 years. Naltrexone was added to Sertraline $100 \mathrm{mg}$ per day which was introduced 2 years before. Within a week of treatment at $50 \mathrm{mg}$ per day of oral Naltrexone, the patient reported a measurable difference in sexual address. He described not being triggered all the time.

\section{THE "OCD-TRACK"}

Cyberaddictions could also be classed among the obsessive-compulsive spectrum disorders. In this case, testing serotonin reuptake inhibitors and possibly antipsychotic would be given priority in investigation.

\section{Available Data}

Atmaca et al. [7] have reported the case of a 23 years old medical school student who complained of excessive Internet use, and was treated by Citalopram. The drug was initiated at a dose of $20 \mathrm{mg}$ /day and subsequently increased to 40 $\mathrm{mg}$ /day within 1 week. After 6 weeks, the Internet-useYBOCS score had only changed from 21 to 18 . At this time, the patient still spent about 33 hours per week in non essential Internet use and reported only little improvement. Subsequently, Quetiapine $50 \mathrm{mg}$ per day was added to the SSRI and titrated up to $200 \mathrm{mg}$ /day within 4 days. Four weeks later, Internet-use-Y-BOCS score was 8, and the patient had reduced his weekly Internet use to 7 hours per week, now reporting to be able to control his use.

Dell' Osso and Altamura [8] have performed a study including 19 subjects meeting study criteria for excessive Internet use receiving open label Citalopram for 10 weeks and then randomized to a 9 week double-blind discontinuation phase. The effect of Citalopram treatment was analyzed regarding (1) hours spent per week in non essential Internet use and (2) with regard to obsessivecompulsive and total scores on the impulsive-compulsive Internet usage disorders Y-BOCS and finally (3) with regard to the clinical global improvement scores. Significant improvement of the non essential Internet hours per week, the modified Y-BOCS and the Clinical Global Improvement (CGI) occurred during the first phase. During the second phase from week 10 to 19 there was no significant difference between placebo and Citalopram.

\section{THE "COMORBIDITY TRACK"}

Cyberaddiction could also be conceptualized as an expression of a different disorder. Cyberaddiction has repeatedly been found to be associated with various psychiatric disorders (cf. Chih-Hung Ko et al., in this issue). According to the hypothesis, cyberaddiction is rather an expression of an underlying psychiatric disorder than a disorder entity itself, the treatment of this underlying disorder should concomitantly alleviate cyberaddiction.

Social anxiety and ADHD are among the psychiatric disorders most frequently associated with cyberaddiction. Social anxiety respectively shyness has been proposed as a predisposing factor for the development of cyberaddiction. In general, comorbidity studies have revealed strong association between social anxiety and the duration of Internet use [9]. Lee et al. [10] investigated 91 male adolescents with excessive Internet use and compared them to 75 healthy comparison subjects regarding prevalence of the short allelic variant of the serotonin principal progene (i.e. 5 HTTLPR). This allelic variant was more frequent in the excessive Internet use group. Excessive Internet users expressing the variant also showed higher harm avoidance and Young Internet addiction scale (IAS) score than those expressing the other serotonin transport gene allele variance.

Similarly, recent studies and demographic reports have noted a high comorbidity of ADHD and Internet addiction $[11-13,14]$. It has been reported that Internet addicted groups have greater ADHD symptoms than non addicted groups and ADHD groups have a greater severity of Internet addiction than the non ADHD groups [12, 15]. Han et al. [11] investigated 62 drug naïve children diagnosed with ADHD and Internet videogame overuse treated with Methylphenidate. After 8 weeks of treatment, IAS scores and Internet usage times were significantly reduced. The changes in the YIAS-K scores between the baseline and 8-week assessments were positively correlated with the changes of ADHD Rating Scale scores as well as omission errors from the Visual Continuous Performance Test.

Despite the good availability of data on comorbidity, the "comorbidity track" until now has not been invested. Two main kinds of studies could be designed: (1) Studies which target specifically cyberaddiction in patients with 
comorbidity, and (2) studies which primarily target the comorbid disorder and besides assess the evolution of Internet activities.

\section{CONCLUSIONS}

On one hand, current data regarding pharmacotherapy of cyberaddiction are meager; on the other hand there is enough rationale to investigate possible treatments. Future research agendas should, nevertheless, pay attention to the diversity of addiction products and of addictive behaviors. e.g., treatment which is efficacious in cybersex addiction must not necessarily be efficacious in gaming addiction, but it could be. Thus, if possible, pharmacotherapeutic research in the field may be tried on one hand to target symptoms which are specific for subclasses of cyberaddiction (e.g. sexual arousal) and on the other hand to address phenomena that are common to all cyberaddictions (possibly to all addictions). Due to the heterogeneity and complexity of the category of cyberaddictions, data helping to set priorities would be helpful. Thus case reports and short case series could be useful to guide research endeavors toward practical objectives. In order to adequately develop pharmacotherapies (and therapies in general), it is, furthermore, essential to use treatment sensible assessment instruments. While currently available scales have primarily been developed aiming at sensibility and construct validity, their sensibility to assess treatment response remains to be ascertained.

Finally, it appears that the still young research on pharmacotherapy of cyberaddiction shares the struggle of the addictions in general to attract the pharmaceutical industry to invest in "de novo developments" and "drug repurposing", and this despite the potential market, highlighting the common stigma of addictive disorders.

\section{ACKNOWLEDGEMENT}

Declared none.

\section{CONFLICT OF INTEREST}

Declared none.

\section{REFERENCES}

[1] Chong CR, Sullivan DJ. New uses for old drugs. Nature 2007; 448 : 645-6.

[2] DiMasi JA, Hansen RW, Grabowski HG. The price of innovation: new estimates of drug development costs. J Health Econ 2003; 22(2): 151 .

[3] Ashburn TT, Thor KB. Drug Repositioning: Identifying and Developing New Uses for existing Drugs. Nat Rev Drug Discov 2004; 3: 673-83.

[4] Koepp MJ, Gunn RN, Lawrence AD, et al. Evidence for striatal dopamine release during a video game. Nature 1998; 393(6682): 266.

[5] Ryback RS. Naltrexone in the treatment of adolescent sexual offenders. J Clin Psychiatry 2004; 65(7): 982-6.

[6] Bostwick JM, Bucci JA. Internet sex addiction treated with Naltrexone. Mayo Clin Proc 2008; 83(2): 226-30.

[7] Atmaca M. A case of problematic internet use successfully treated with an SSRI-antipsychotic combination. Prog Neuropsychopharmacol Biol Psychiatry 2007; 31(4): 961-2.

[8] Dell' Osso B, Altamura AC, Hadley SJ, Baker BR, Hollander E. An open-label trial of escitalopram in the treatment of impulsivecompulsive internet usage disorder. Eur Neuropsychopharm 2006; 16(Suppl 1): S82.

[9] Wolfling K, Buhler M, Lemenager T, Morsen C, Mann K. Glücksspiel- und Internetsucht. Nervenarzt 2009; 80(9): 1030-9.

[10] Lee YS, Han DH, Yang KC, et al. Depression like characteristics of 5HTTLPR polymorphism and temperament in excessive internet users. J Affect Disord 2008; 109(1-2): 165-9.

[11] Han DH, Lee YS, Na C, et al. The effect of methylphenidate on Internet video game play in children with attentiondeficit/hyperactivity disorder. Compr Psychiatry 2009; 50(3): 2516.

[12] Yoo HJ, Cho SC, Ha J, et al. Attention deficit hyperactivity symptoms and internet addiction. Psychiatry Clin Neurosci 2004; 58(5): 487-94.

[13] Yen JY, Ko CH, Yen CF, Wu HY, Yang MJ. The comorbid psychiatric symptoms of Internet addiction: attention deficit and hyperactivity disorder (ADHD), depression, social phobia, and hostility. J Adolesc Health 2007; 41(1): 93-8.

[14] Chan PA, Rabinowitz T. A cross-sectional analysis of video games and attention deficit hyperactivity disorder symptoms in adolescents. Ann Gen Psychiatry 2006; 5: 16.

[15] Ha JH, Yoo HJ, Cho IH, Chin B, Shin D, Kim JH. Psychiatric comorbidity assessed in Korean children and adolescents who screen positive for Internet addiction. J Clin Psychiatry 2006; 67(5): 821-6. 